\title{
A DECISION-MAKING PROCESS FOR PLANNING PATIENT EDUCATION
}

LINDA K. STRODTMAN, R.N., M.S.

Medical Nursing Service and School of Nursing, University of Michigan,

Ann Arbor, Michigan

\begin{abstract}
Health professionals tend to resist incorporating education into their patient-care activities despite increasing evidence that educating patients in self-care management can favorably influence health outcomes. Moreover, health professionals often lack skill and expertise in developing patient-education plans. To remedy this situation a new method was developed for teaching health professionals how to integrate patient education into the patient's plan of care. Using the guided design approach, a project was adapted to teach health professionals how to plan patient education for the person with diabetes mellitus. In this method health professionals work as members of a team to address a particular patient's educational needs in a real-life situation.

More than 250 health professionals have participated in a two-hour workshop utilizing this project. It provides participants with a systematic approach for planning patient education and gives them practice in writing an individualized patient-education plan that takes into consideration the patient's overall needs and goals. This instructional method not only has helped health professionals differentiate patient goals (outcomes) from health professional actions (process) but also has provided health professionals with the opportunity to learn how to work together. The benefit of the "team experience" was the one participants most often identified as having the greatest value.

This paper includes a description of the project, a copy of the guided design tool, and specific instructions for its use to enable others to try this method.
\end{abstract}

\section{INTRODUCTION}

Patient education is increasingly being recognized as an integral part of quality health care. Several key events have contributed to this development. The parallel increase in prevalence of chronic disease and in age of the population requires people to engage in new patterns of care over a longer lifespan. Thus more attention must be given to helping people care for themselves. Consumers' movements have alerted people as to their rights to receive health care, to be informed about their care, and to participate in care decisions. Patients are coming to recognize the value of this to themselves, and health professionals are coming to recognize their obligation to educate patients. The legal system has highlighted the need for "informed consent," and the relationship of improved communication between the patient and health professional to the reduction of malpractice litigation. ${ }^{1}$ The cost of health care has become a nationwide concern, particularly when studies have shown patient compliance rates with medically prescribed regimens frequently to be no higher than $50 \% .^{2}$ Research has shown that when patients are adequately informed and included in the educational process concerning their own care and treatment, the care costs decrease, patient and family well-being increase, and compliance with the therapeutic regimen improves. ${ }^{3,4}$ As our health-care financing system moves into prospective reimbursement, such as with the diagnostic related groups, the emphasis will shift to shortening hospital stays, thereby creating even greater demands for effective patient education.

Despite the importance of patient education and the continuing trend among hospitals to support patienteducation activities, those engaged in directing or developing patient-education programs still face the problem of motivating health professionals to teach their patients. The patient's education should be a planned experience that is individualized to one's needs, abilities, and personal resources. ${ }^{5}$ This is different from the inconsistent teaching efforts that occur in many clinical practice settings. Unplanned or spur-of-the-moment experiences frequently occur when health professionals "have the time," or they are incidental to the provision of other medically oriented therapy such as giving medication. Planned patient education is also different from the sporadic and often incomplete education that occurs when patients are sent to a class, given booklets, asked to tune into a hospital television channel for bedside viewing of a health-related program, or placed in front of a computer terminal and given directions for learning about their disease and its treatment.

While each of these educational approaches has merit, they are only methods of teaching. The more 
critical process in patient education, and the one most often neglected by health professionals, is the decision-making process that involves assessing the patient and establishing a written, individualized plan that takes into consideration the patient's overall needs and goals. This paper describes one method for teaching health professionals these crucial clinical decisionmaking skills.

\section{HISTORICAL BACKGROUND}

In 1977 the University of Michigan received funding for a diabetes research and training center (DRTC). Part of this grant was devoted to the establishment of a model demonstration unit, the Diabetes Center Unit, which has as one of its aims the education and training of health professionals in state-of-the-art patient care in diabetes.

Since evidence indicates that appropriate self-care can enhance the regulation of diabetes mellitus and ameliorate its morbidity, a self-care program was developed for this inpatient unit..$^{6-8} \mathrm{~A}$ vigorous patient-education program was planned, in which the patients learn basic health-care practices, collect and monitor information related to their own health status, and modify certain self-care practices that adversely affect their health.

Enhancing the effectiveness of health professionals as teachers became a major focus of the unit. Two approaches could have been used. One approach would have been to invest the teaching function in one or two health professionals and have them be the patient educators for the unit. A key problem with this approach is that the other care providers abdicate their healtheducation responsibilities to the educators, who then become too overworked to deliver individualized education to each patient. A second method, and the one chosen, was to teach all members of the care team to incorporate health education as part of their overall patient-care responsibility.

Another vital component of the unit's patienteducation program was the establishment of an interdisciplinary team approach to patient care. When patients are admitted to the unit, they are assessed by each of the primary health-care team members (dietitian, nurse, physician). From these assessments the patient's health-management strengths and deficits are identified and agreed upon by the whole team at a twice-weekly team-care conference. Health-management deficits are defined as the inability to manage aspects of one's own treatment or health-promotion activities because of cognitive, psychosocial, or physiological limitations. ${ }^{9}$ In collaboration with the patient and family, a healtheducation plan is then developed, which becomes an integral part of the patient's total health-care management. Depending upon the patient's learning needs, interests, and capabilities, specific educational interventions are offered (Table I).
Table I. Repertoire of patient-education interventions in the Diabetes Center Unit.

One-to-one instruction from specific health-care team members

Printed materials (single-concept monographs, books, journals)

Formal group sessions (a weekly 12-session series)

Support group discussions

Peer interaction among patients

The patients' progress with their prescribed care program is documented in their health-care records utilizing an interdisciplinary problem-oriented approach. Retrospective audits of these charts have consistently indicated a high level of care for these patients. The patients' level of self-care knowledge, as measured by tests given on admission and at discharge, has significantly increased and their reported level of satisfaction with care also has been high.

An important activity has been to incorporate this patient-care and educational approach into the professional education and training programs of the DRTC. Recurring educational symposia (two to three days each) for practicing health professionals from community settings have been established as one mechanism. The symposia provide health professionals with current information about diabetes and diabetes care. Each symposium has included a workshop devoted to patient education.

\section{WHY GUIDED DESIGN}

Traditional methods of teaching health professionals how to teach often focus only on the theoretical aspects of the teaching-learning process. Frequently little has been offered to help health professionals transfer this information to their own practice setting. Another problem is that patient education is not given a value equal to that of other aspects of care and treatment. One reason cited is that the health professional does not have enough time. When care demands increase, patient education is one of the first aspects of care to be dropped. Our experience suggests that this need not be the case. The problem may be more a lack of commitment to teaching than a true lack of time. In our unit, even when staffing was markedly reduced, chart audits indicated that patient education and other elements of care were maintained at a high level, as was the amount of staff participation in the unit's formal patient-education activities. Since patient education is a high priority of the unit's leaders (head nurse, and assistant head nurse), this expectation is likewise transferred to the staff.

For all these reasons, it was important to find a method of teaching professionals that could help them 
incorporate patient education into their daily practice. Since the provision of patient education requires the health professional to have expertise in decision making, the decision-making model of guided design was selected. ${ }^{10}$ Other reasons for selecting the guided design approach were:

1. To facilitate group process skills that are needed in an interdisciplinary team approach to patient management

2. To incorporate a problem-solving approach that is similar to the care-planning process that most nurses are taught (we anticipated the majority of symposia participants to be nurses)

3. To use a method that could easily be adapted for the content we wanted to teach and one that could be used in a limited amount of time

4. To give participants an opportunity to apply content to a typical practice situation

\section{DESCRIPTION OF THE GUIDED DECISION-MAKING MODEL}

Guided design is an educational strategy initially developed by Charles E. Wales and Robert A. Stager for engineering students. It has subsequently been used by many educators from a variety of disciplines. The key elements of this method that make it so successful are: (1) it teaches subject content by using a step-by-step feedback process in which the participant is guided through a series of instructions for action and follow-up evaluation of their work; (2) it helps participants learn how to make decisions; (3) it permits the application of subject matter to real-world problems; and (4) it provides an organized group experience in which participants can practice interaction skills.

Using the "Fishing Trip" format from Wales and Stager, ${ }^{10}$ we developed a guided design project for use in the Diabetes Care Symposia (Appendix). Table II lists the objectives for this two-hour workshop.

The workshop begins with an individual exercise in which each participant is given assessment data about a patient admitted to a hospital unit. The patient is a middle-aged man with newly discovered diabetes mellitus. $\mathrm{He}$ also has several other health problems, living habits, and psychosocial factors that require consideration in the planning of his self-care management. Each participant is asked to rank independently a list of resources according to their appropriateness for the patient. These include a film on coping with stress, oneto-one instruction, a series of patient-education classes, family diet counseling, visiting nurse referral, and so forth.

Next the workshop participants are divided into groups of five to eight people with instruction to function as the patient's health-care team. During this part
Table II. Planned patient-education workshop objectives.

Given a patient situation and planning process, the participant will be able to:

1. Devise an appropriate educational plan

2. Describe the five steps of the patient-education process:
A. Identification of the educational needs of the patient and family
B. Establishment of educational goals
C. Selection of appropriate educational methods
D. Implementation of the educational plan
E. Evaluation

3. Identify some practical techniques and methods to be used in facilitating patient and family education about self-care

of the project the participants are led through a series of "instruction" exercises with subsequent "feedback" on their actions. The group begins by discussing the patient and what to do for him educationally. In the first feedback step, the participants are given a list of actions that are likely to be similar to the ones their group proposed. Then it is pointed out that these items are possible solutions only and that the patient's problems have to be identified first. This is the typical initial course of action that health professionals take: proposing actions without first identifying the patient's problems. With chronic diseases this is of particular concern because there are many standard methods of treatment that health professionals fall into the routine of prescribing without considering the individual needs of the patient. The group is then led through the decision-making process of identifying the patient's problems, establishing priorities, formulating goals, selecting an appropriate educational intervention, implementing the intervention, and evaluating the results.

Throughout the exercise the participants are given feedback about their decisions by a variety of team members (dietitian, nurse, physician, and social worker) so that each person learns about the roles of different health professionals. Prior to the evaluation step the participants again rank the available resources but this time as a group. In the evaluation step the group is given advice from a fictitious well-known diabetes educator about the patient's educational care plan, and they compare their plans individually and in their group with the "expert." Also included in the symposium syllabus for the participants are tools for use in educating patients, ie, diabetes knowledge tests, self-care assessment questionnaire, patient-education booklets, and list of available audiovisual media. At the end of the planned patient-education session the course instructor discusses the process, answers questions, and asks for feedback from the participants about the project. 


\section{RESULTS}

More than 250 health professionals have participated in this guided design project. About $50 \%$ of the participants were registered nurses, $30 \%$ dietitians, and the remaining $20 \%$ social workers, pharmacists, and physicians. The participants were from hospitals, clinics, private practice offices, nursing homes, and health departments.

In the postcourse evaluation the participants have consistently described these sessions as beneficial, with written comments such as "found working with team stimulating," "worthwhile for those of us who don't have team in own hospital," "feedback system excellent," "much personal insight gained," "learned benefits of group consideration, differing priorities, and need to establish plan," "team worked well together," and "good demonstration of team approach."

The most frequent benefit cited has been the team approach. This was surprising because it was not the primary purpose of the session when it was developed. The instructors' observations of the workshop participants have supported this finding. Evidently, for many of the workshop participants, it was their first time working as a team with members of other health-care disciplines. This was particularly true for those groups that had physicians as team members. Because only a limited number of physicians attended these conferences, only a few groups had physician team members. This exercise seemed to be highly beneficial for the physician members, as many commented that they had a much better understanding of the roles of the other team members as a result of this exercise.

The group dynamics were varied. Some groups readily chose a leader and worked through the decision-making process smoothly, whereas members of other groups encountered difficulty in working together. An instructor was available to give assistance to each group when needed. All of the groups completed the decision-making process, but a few found it difficult. One such group could not reach consensus and actually halted the process. When it was recognized that they might need help from the instructor, they proceeded to solve their own problem without assistance. Another participant commented on this event in the course evaluation as follows: "I would like to have more guidance from one of the staff. If your group has an outspoken person, you get very little done. Maybe this in itself is a learning experience for dealing with a team."

Other observations were that many health professionals are not confident about making decisions. This was reflected in comments about wanting to know if their group decisions were "right" or if they had the "right" answers, despite the fact that participants were told initially that there were no right or wrong answers. Also, many of the health professionals had difficulty in distinguishing health-professional activities or processes from patient goals or outcomes. This latter observation may be particularly important. The fact that the health professional cannot identify the goal for the patient may be a reason that patient education is often ineffective. Also this speaks to the necessity of having an individualized patient-education plan that is modified according to the patient's response.

On the course evaluation, participants were asked the question, "Will you do anything differently in your practice as a result of attending this course?" Replies included "team effort," "respect and appreciate to a fuller degree patients' problems and concerns," "plan for common objectives and goals and allow more time for meeting patient needs," "gear teaching to individual need," "emphasize need for patient inclusion as part of health-care team," "be assertive with medical staff," and "plan education differently."

\section{DISCUSSION AND GUIDELINES FOR CONDUCTING SESSION}

Since the original project was designed, the tool has undergone two revisions. ${ }^{11}$ These modifications were primarily the result of advances in diabetes care. For example, self-monitoring of capillary blood glucose was added. The tool is best used in a two-hour workshop, but it can be modified for one-hour sessions by eliminating the beginning exercise.

Health-professional participants are assigned to small groups. From the experience of the session leaders, a group of five to six people seems to function most effectively. Attempts should be made to have a variety of disciplines represented in each group.

At least two workshop facilitators are recommended for each 50 participants (ten groups of five). The role of the workshop facilitators is to introduce the topic, explain the format (series of instructions with feedback in a stepwise progression), and give each feedback and instruction set to the groups as they become ready for them. The facilitators, besides being available for questions, interact with the groups to help resolve problems so that each group completes the total exercise within the time period. Adherence to the exact times is not important as long as each group can complete the entire project. Some groups spend longer with some portions than others. Most of the time spent by the facilitator with each group will be directed toward the groups' "team" functioning. This will depend to some extent on how many health-care disciplines are represented within each group and how familiar they are with each other's roles.

After each group has finished the project a facilitator reviews the evaluation section and the summary of the steps completed. The session concludes with discussion (led by the group leaders) as to how each group functioned, followed by their evaluations of the method. 
Another group of health professionals in educator roles has also successfully used this guided design tool. ${ }^{12}$ They modified the patient situations to reflect the type of patients and care needs seen in their setting. Because of their success and ours, we invite others to try this method. It can be adapted to patient populations other than those with diabetes. We believe that this method has considerable advantage over other ways of teaching patient-education skills, and we would like to hear about the experience of others with this nontraditional approach.

\section{ACKNOWLEDGMENTS}

This project was one component of Diabetes Care Symposia sponsored by the Michigan Diabetes Research and Training Center, University of Michigan, Ann Arbor, Michigan, under NIH grant 2 P60AM20572-06. The author thanks Jill J. Quigley, former assistant head nurse of the Diabetes Center Unit, for coauthoring the initial guided-design tool.

\section{REFERENCES}

1. Simonds SK. Patient education: The next ten years. In: Rx: $E d u$ cation for the patient. Institute proceedings. Carbondale, Illinois: Department of Health Education, Southern Illinois University, 1975:112.

2. Rheingold JJ. Patient compliance. In: Rx: Education for the patient. Institute proceedings. Carbondale, Illinois: Department of Health Education, Southem Illinois University, 1975:65.

3. Task Force on Patient Education for the President's Committee on Health Education. The concept of planned, hospital-based patient education programs. Health Educ Monogr 1974; 2(1):7.

4. Mazzuca SA. Does patient education in chronic disease have therapeutic value? J Chronic Dis 1982; 35:525.

5. Davis WK, Hull AL, Boutaugh ML. Factors affecting the educational diagnosis of diabetic patients. Diabetes Care 1981; 4(2):275.

6. Miller LV, Goldstein J. More efficient care of diabetic patients in a county hospital setting. $N$ Engl $J$ Med 1972; 286:1388-1391.
7. Peterson CM, Forham SE, Jones RL. Self-management: An approach to patients with insulin-dependent diabetes mellitus. Diabetes Care 1980; 3(1):82-87.

8. Geller J, Butler K. Study of educational deficits as the cause of hospital admission for diabetes mellitus in a community hospital. Diabetes Care 1981; 4(4):487-489.

9. Gordon M. Manual of nursing diagnosis. New York: McGrawHill Book Co, 1982:36.

10. Wales CE, Stager RA. Guided design. Morgantown, West Virginia: Center for Guided Design, University of West Virginia, 1977.

11. Quigley JJ, Strodtman LK. Guided decision-making in planning patient education. Unpublished manuscript. Medical Nursing Service, and Michigan Diabetes Research and Training Center, The University of Michigan, Ann Arbor, Michigan, February, 1980.

12. Ballard T, Kelleher K, Burns D. Diabetes in the 1980s. Workshop sponsored by Veteran's Administration Hospital, Seattle, Washington, 1980 .

\section{APPENDIX}

\section{Guided Design Tool}

Note for Instructors: Give the participants the various sections of the tool in the following order:

\section{Section}

1. First Exercise

2. Second Exercise Instruction A

3. Feedback $A$ Instruction B

4. Feedback B Instruction $\mathrm{C}$

5. Feedback $C$ Instruction D

6. Feedback D Instruction E

7. Feedback $E$ Instruction $\mathrm{F}$ Summary

\begin{tabular}{c} 
Approximate Time \\
\hline 15 minutes \\
10 minutes \\
10 minutes \\
10 minutes \\
10 minutes \\
10 minutes \\
20 minutes
\end{tabular}

Approximate Time

10 minutes

10 minutes

10 minutes

\section{FIRST EXERCISE: INDIVIDUAL WORK}

\section{Introduction}

Every health professional is involved in patient educationsometimes formally, sometimes informally. Informal education may include telling a patient the name of a medication as you administer it or helping a patient select appropriate food from a hospital menu. This project involves learning and practicing a more formal approach for planning the patient's educational experience. You will be asked to participate in this project by working as part of an imaginary health-care team, but first read the following information so that you can become familiar with the setting and your patient.

\section{The Setting}

Mr. Robert Benito is admitted to your inpatient hospital unit. He tells you, "I don't know why I'm here, I feel just fine." He describes going to see his usual health-care provider at a community clinic for routine blood pressure monitoring earlier in the day and then being told to go immediately to the hospital because he "had high sugar."

His admission nursing assessment reveals: 


\section{Patient Profle-Health Management Status}

A 42-year-old, well-dressed, Italian-American male who is pacing the room. He doesn't understand why he has to be admitted. He desires "to get out of here as quickly as possible." Admitting medical diagnosis-type II noninsulin dependent diabetes mellitus (NIDDM). (Note: A person with type II diabetes may or may not require insulin therapy.)

Social Status: Married, father of three children, ages 5, 8, and 11 years. Lives with wife and children in ranchstyle home. Employed as janitor in elementary school. Wife works as nurse's aide in community hospital. Hobbies--fishing and bowling on weekends. Catholic religion. Youngest of 19 children (10 of whom still live in Italy). Financial status-adequate; "not rich but we get by." BC/BS insurance-does not pay for medication or health supplies.

Energy Balance (Nutrition/Physical Activity/Hypoglycemic Agents): Has been eating nonrestricted diet. Admission weight $235 \mathrm{lbs}$. Height $5^{\prime} 7^{\prime \prime}$. Lost $25 \mathrm{lbs}$ in last two weeks without dieting; weighed 160 lbs at age 26. States, "I know I need to lose weight, but I like food." No specific food dislikes; eats when he's hungry-one to two meals a day. Wife prepares meals except on fishing trips. Stopped drinking alcohol two years ago when told he must due to "liver problem." Has own teeth, none missing, brushes teeth one or two times a day. Sees dentist once a year. No previous experience with taking insulin. States his mother and one brother are diabetic but have never followed a diet or taken insulin. Works full-time, mostly standing, walking, sweeping, etc. Some heavy lifting on occasion. Sedentary activity on nonwork time except for weekly Friday night bowling.

Elimination Status: Denies constipation or diarrhea. No UTIs; no difficulties with urine stream or force; c/o frequent urination-three or four times during night, 22 times in one day. Adm. urine glucose $3 \%$ (Testape) neg. ketones (Ketostix).

Skin Status: No rashes, bruises, lesions noted. Dark complexion. Mouth "dry." Asking for water. Diaphoretic brow and palms. Toenails cut short. Dry skin on feet-uses lanolin lotion. Feet pink, warm with strong pedal pulses, no callouses or lesions.

Respiratory Status: Smokes ppd $\times 15-20$ years; denies past respiratory disease; no productive cough or DOE.

Circulatory Status: Adm. BP 140/80, pulse, 90 lying; $138 / 82$, pulse, 92 standing. Regular pulse. No edema noted. Occasionally feels bloated. Told that he was hypertensive $1 \frac{11}{2}$ years ago; taking hydrochlorothiazide $50 \mathrm{mg}$ daily.

Motor Ability Status: Ambulatory, works as school janitor without limitation in lifting or movement. Works from 6:00 AM to 2:30 PM; noted increased fatigue over last several weeks. During interview shifted position often; tremor noted when reaching for water pitcher.

Body Temperature Status: $98.8^{\circ} \mathrm{F}$ oral on adm. No history of recent fever or infection. Gets a cold about once a year.

Reproductive Status: Sexually active, satisfied with sexual relations. States no problems.
Physical Rest and Comfort Status: Amount of sleep varies greatly, three to eight hours/night. No difficulty sleeping except during recent episodes of nocturia.

Cognitive Perception: C/o blurred vision for two weeks, able to read admission knowledge test. Occasional numbness in hands described as "pins and needles." Speaks with heavy accent. Simple nonmedical vocabulary. Knowledge pretest score five correct answers out of 38 questions. Missed items in all areas of diabetes selfcare. Unable to say how he learns best; seventh-grade education. States, "You'll probably have to tell me everything." Insists that he is not sick and has never been ill.

Emotional Status: States he and his family get along well. States none of other family members who have diabetes take insulin, "But the doctor says I will have to." Rapid speech, asking many questions, easily distracted. Keeps repeating, "I feel fine-they say I have high sugar but I don't think I have it." When pressed, admits to polyuria, polydipsia, polyphagia, dry mouth, and blurred vision for several weeks. Wife finally insisted he see local MD to have BP checked. "He (doctor) told me I couldn't even go home; I had to come here immediately." Serum glucose 468 on admission, after eating an orange and drinking a coke three hours previously. States his wife is very supportive and "I leave a lot of things for her to take care of." States that he "likes to talk things out when he has questions."

\section{Ranking of Resources}

Just from reading this information you probably have some ideas about how Mr. Benito might be helped. The following resources are available in your health-care setting. You decide to rank these resources according to their appropriateness for Mr. Benito. Independently rank the following list of resources. Mark a \#1 next to the most valuable resource, \#2 for the next most valuable resource, \#3 the next, etc; continue to \#17 which is the least valuable resource. Write your list of numbers in the column marked I. (See Chart A.) Do not take more than five minutes for this activity. (Disregard the other columns for the time being.)

\section{SECOND EXERCISE: TEAM DECISION-MAKING}

Form groups of four to eight people. This group is to function as a health-care team.

\section{Introduction}

The material you are about to use is concerned with planning patient education. This material is organized in an "instructional-feedback" pattern so that you can actively participate in the planning of an educational program. Each instruction is a question or a problem which will guide you through the basic steps in developing an educational plan. Each feedback* summarizes the discussion you probably had

*The purpose of the printed feedback is to allow us to compare your reasoning to that of other people. You should not feel that you must accept any of these decisions. 


\section{Chart A}

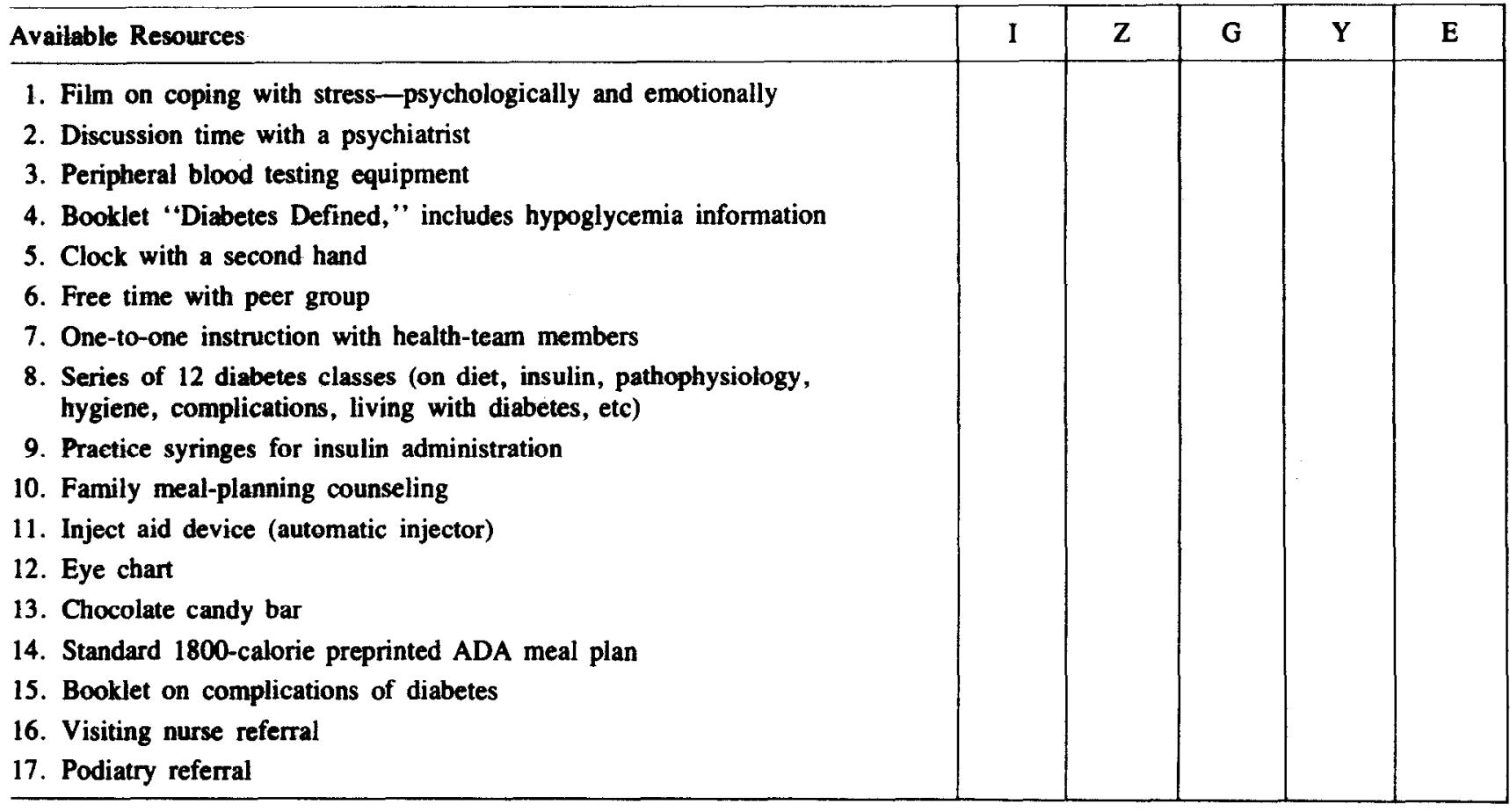

when responding to the instruction. Read each "instruction," discuss it with your team, and decide on a group response. Take your team's written response (you probably need to appoint a secretary to record your group decisions) to the instructor. If the instructor believes the team's work is complete, you will be given a copy of the feedback and the next instruction. If work is not yet complete, the instructor will ask questions to stimulate further thinking.

\section{Instruction A: The Problem}

The next day you ask that $\mathrm{Mr}$. Benito be discussed in the patient-care conference. The dietitian, pharmacist, physician, social worker, and primary nurse are present and have assessed the patient through either interviews, questionnaires, and/or pretests. You begin the discussion saying, "Let's talk about what we are going to do educationally for Mr. Benito."

Assume you are a member of the health-care team. Briefly discuss with your group what you would do to meet $\mathbf{M r}$. Benito's educational needs. Allow five minutes for this activity. When finished, contact the instructor.

\section{Feedback A}

The team had quite a discussion about what to do for Mr. Benito. Some of their ideas were to:

1. Send him and his wife to all classes, particularly the class on insulin

2. Give him booklets on diabetes

3. Show him a film on hypertension

4. Teach him about managing his diet while on fishing trips

5. Tell him the importance of regular hypertension monitoring
6. Show his family the film "What is Diabetes"

7. Give him the address of a local "stop smoking" support group

8. Teach him about the genetics of diabetes

9. Teach him about the diabetic complications he can minimize or avoid by following the care regimen

\section{Instruction B: Identify the Problems}

Looking at the lengthy list they had compiled, the social worker said, "I think we have a lot of good ideas about possible solutions or what to do, but we've been talking about how to solve problems we haven't even identified yet. Let's see if we can decide on some problems that need to be worked on."

The team agreed and they proceeded with this task. list.

In your group, proceed with developing a patient problem

Take no more than ten minutes for this activity. When finished, contact the instructor.

\section{Feedback $B$}

This time the team developed a list of what they thought were Mr. Benito's health-care problems. It included:

1. Obesity

2. Moderate anxiety related to inability to accept diagnosis and hospitalization

3. History of excessive alcohol intake

4. Blurred vision

5. Lack of knowledge in diabetes self-care in the following areas:

a. Medication

b. Diet 
c. Exercise

d. Blood testing

e. Foot care

f. Complications

g. Hypoglycemia

h. Hyperglycemia

i. Follow-up care

6. History of hypertension that requires continued monitoring

7. Variable sleep pattern

8. Heavy cigarette-smoking history

\section{Instruction C: Establish the Priorities}

The physician says, "I think we've listed too many problems. How many of these can we realistically do something about during this five- to seven-day admission? Let's put the problems in priority and work on them one at a time."

In your group, list the above problems in priority order according to those that your team thinks are most important. Spend approximately five minutes on this activity. Contact the instructor when you are finished.

\section{Feedback $C$}

After a brief discussion the group decided on the following priorities

1. Lack of knowledge and skill in diabetes self-care management
a. Diet
b. Medication
c. Hypoglycemia
d. Blood testing
e. Follow-up care
It was decided that these are interrelated and must be considered as primary concerns.

2. Moderate anxiety

3. Obesity

The other problems were agreed to be secondary to assuring initiation of safe diabetes self-care. They will be addressed after the primary problems are resolved.

\section{Instruction D: Facts, Assumptions,} Constraints-Choose a Solution

"Before we decide upon goals and actions, I have some information that I think will make an impact on how we establish our plan," the pharmacist said. "Mr. Benito takes his hydrochlorothiazide only when he feels bloated. He was also prescribed a low-salt diet, but he doesn't follow it. His wife usually has to coerce him into keeping his routine hypertension checkup. I think his past health compliance should be considered-it seems that he is motivated by concrete symptoms and by his wife."

The social worker related a situation which also reflects $\mathrm{Mr}$. Benito's family attitude about hospitals and health. While having routine surgery, Mr. Benito's father was found to have cancer. He died soon after discharge. The family continues to insist that had the father not entered the hospital, he would be alive today. The family subsequently switched their healthcare to another hospital. "I see similarities to this in $\mathrm{Mr}$. Benito's statement that he would be 'okay' today if he hadn't let his wife talk him into seeing his doctor."
The dietitian shared information from her diet history. "He eats a 'typical' starch diet-many pastas, breads, and rich pastries. Mr. Benito attempted to diet in the past, but his family laughed at his 'rabbit food' so he ate alone and soon stopped dieting. I think his family has a strong influence on his behavior."

The physician related that $\mathrm{Mr}$. Benito sees most of his extended family socially but that a portion of his family is still in Italy and do not speak English. "I wonder if there are cultural influences on his family or personal interactions that we should be aware of?'

The dietitian says that Mr. Benito bowls Friday nights and then goes fishing literally all the rest of the weekend, namely from 3:00 AM to 10:00 PM Saturday and Sunday and rarely does any other exercise. "I can calculate snacks for these activities or a special weekend diet and thus avoid the need for him to learn calculations for exercise snacks."

The primary nurse added that Mrs. Benito works as a nurse's aide in an area hospital. "Since she works she will be unable to attend classes during the day. However, she may have some basic health knowledge which we could build upon and use." The nurse described his pushed speech, easy distractability, anger, complaints of blurred vision, and a general bad feeling after attending a class on diabetic complications. "I think we need to be very selective in the classes we send him to. He becomes very anxious when given too much information. He needs just the basics for now."

The social worker summed up the discussion. "Now that we know some specific considerations for this patient, let's write out our goals and how we want to achieve them."

Write out goals and actions for just one of the problems that involves patient education as listed in Feedback C. Take about ten minutes for this activity (see Chart B). When finished, contact the instructor for feedback.

Chart B

Problem \#

Expected Outcome (goal) Plan (action)

\section{Feedback $D$}

Chart $\mathrm{C}$ (below) itemizes various goals and actions to be considered for Mr. Benito.

\section{Instruction E: Analysis and Synthesis-} Select Appropriate Educational Method

"Now that we have developed our plan, I'd like for us to rank the educational resources we should use. Since we know what we want to accomplish, this should be a snap," the nurse said.

Discuss the items in the Available Resources list in the Ranking of Resources section of the First Exercise and rank 


\section{Chart C}

Problem \# 1. Lack of knowledge re: DM self-care management

\section{Expected Outcome}

A. Diet

Patient/significant other (pt./SO) will correctly plan sample home menu using exchanges. $\mathrm{Pt}$. will correctly select fonds from daily menu using meal plan.

B. Medication (insulin)

Pt./SO will correctly administer insulin $\times 3$ days (includes skill and site rotation).

C. Hypoglycemia

Pt. will state usual or his own signs and symptoms of hypoglycemia and how to treat hypoglycemia.

\section{Blood glucose monitoring}

Pt. will correctly test his blood glucose level using a peripheral glucose monitoring method.

E. Follow-up care

Pt. will state plans for follow-up care. Pt. will state when to contact care team.

\section{Plan}

- Stress basic diet concepts-avoid exercise calculations, restaurant menus, etc.

- Send to meal-planning classes.

- Review class content every evening after class.

- Include wife in diet instruction in the evening.

- Nurse to draw up first injection, pt. to give first injection tomorrow. After mastery of insulin injection, then go on to preparation of insulin dose.

- Give patient-education booklet on insulin.

- Review perception of class content after class.

- Individual instruction regarding signs and symptoms and treatment of hypoglycemia.

- Give booklet with written lists of signs, symptoms, and treatment.

- Validate with him by use of a peripheral glucose monitoring technique his actual blood glucose when and if he has symptoms of hypoglycemia.

- Have pt. observe peripheral blood glucose monitoring initially. Gradually, have him take over doing it after he has mastered each step.

- Show him different types of supplies and equipment. Help him choose a method that is compatible with his abilities and finances.

- Review signs and symptoms and home test results that would indicate a need to contact his health-care provider.

- Give him information about classes and class times so that he can continue with education on an outpatient basis.

- Give him unit telephone number in case he has questions before his next visit with his health-care team.

- Discuss with him the possible need for a follow-up public health nurse visit.

Problem \#2. Moderate anxiety related to diagnosis and hospitalization

\section{Expected Outcome}

Pt. will verbalize concerns to family and staff. Anxiety will decrease to the point that patient can (1) learn and (2) perform basic self-care.
- Note anxiety behaviors.

- Chart anxiety-producing situations.

- Encourage him to talk. Experiment with ways to decrease his anxiety (distractions, talking with family or other patients, learning more about diabetes).

- Encourage pt. to interact with other patients.

- Reassure pt. regarding "normalcy" of fears.

- Minimize anxiety's interference with learning by using mostly one-to-one instruction.

- Expect need for much reinforcement in teaching.

- Show patient and family film on "Understanding Stresses and Strains," a cartoon film that uses a humorous, light approach in discussing life stresses, and discuss it with them afterwards. 


\section{Chart C (Continued)}

Problem \#3. Obesity

Expected Outcome

Pt. will lose weight while hospitalized. Pt. will state plan to continue weight loss. Pt. will state methods to modify past eating habits.
Plan

- Determine past eating habits/patterns-especially emotional and cultural influences.

- Determine family's attitude regarding "acceptable" food.

- Initiate session with patient, family dietitian and nurse to problem-solve.

- Explore alternatives to present eating habits. these items with your group. Record the number representing the group's decision in column $G$ (in other words, all members of your group will record the same number in column $\mathrm{G}$ ). Do not change your previous rating in column I. Try to do this activity in approximately ten minutes.

\section{Feedback $E$}

The result of this instruction is your ranked list of items.

\section{Instruction F: Evaluation}

The team discussed their plan with the patient and his family. They were enthusiastic about it. With their acceptance the initial plan was implemented. The team found that as the patient progressed educationally and as they learned more about him as an individual, their plan also needed constant updating.

A diabetes educator from the state affiliation of the American Diabetes Association visits your hospital and presents a workshop on education of the patient with diabetes. In a small-group presentation, you present the process your team uses to develop an educational plan. As an evaluation of your method and as a learning experience for your team, you ask the guest speaker to review the data and comment on the plan and the methods for education and management of this patient and his family. She also ranks the educational resources. Her selections and rationale for these selections are as follows (transfer these numbers to column $\mathrm{E}$ of the Available Resources list):

(1) One-to-One Instruction with the Health Team Members. In an anxious patient, you need to minimize extraneous stimuli. You can best control content and check immediately for comprehension on a one-to-one basis. In other words, you can tailor the words and concepts to allay the patient's anxiety and still meet his learning needs.

(2) Family Meal-Planning Counseling. This is a high priority because it influences diabetes regulation and obesity. A change in diet always affects the entire family-the person doing the cooking should always be included in diet instruction, and others should be included to gain an understanding of why the family member is on a special diet, and thus elicit support. In this case, the counseling sessions could enlighten the team to the influence of culture and ethnic group on eating habits, eating patterns to be reinforced, and behaviors which are amenable to alterations. This is particularly important for this patient since he and his family eat many foods related to their ethnic heritage, ie, pastas, casseroles, etc. His meal plan should show him how to incorporate some of these foods.

(3) Booklet "Diabetes Defined,' Which Includes Information on Hypoglycemia. In a newly diagnosed diabetic, fear regarding his ability to recognize hypoglycemia is common. Although some anxiety is a motivator, in an overly anxious patient a verbal listing of symptoms may not be retained. Written material is useful for home reference but should not be relied upon for the total instruction. Written material without verbal clarification may only increase anxiety. The rest of the information in the booklet about what diabetes is-particularly type II diabetes-will be useful in the one-to-one instruction. Even though the material is presented at a basic level the patient may not be ready to hear the details. Often we have a need to see that a patient understands his disease when $h e$ is most concerned with what he must do, how he must do it, and when. However, depending on his progress and our ability, he may show a readiness for this at some point during his hospitalization or after he goes home. In the latter case he will have the booklet information available. Point out the important information for him by underlining it or circling the page numbers.

(4) Practice Syringes for Insulin Administration. These are useful tools to increase the patient's confidence in preparing his insulin dosage. Undoubtedly, insulin administration is a major hurdle for the person with newly diagnosed diabetes. When practice equipment is used, remember to use bacteriostatic water or saline rather than insulin and assist the patient during initial practice. Although you may delay having the patient prepare this insulin dose it is usually best to have the patient give his very first injection of insulin. (Prepare the dose for him.) (5) Film on "Coping with Stress-Psychologically and Emotionally." This film title sounds beneficial for this patient-perhaps it associates stress with its influence on blood sugar and blood pressure. This patient definitely needs to hear that we all have stress but that how we deal with it may make a difference in our health. It's also good to include the family in this activity since they can be very supportive if they are involved.

(6) Free Time with Peer Group. Group milieu can be very instructional and supportive. Hearing life experiences of others with diabetes can be reassuring to a person with a new diabetes diagnosis. However, consider your group carefully before encouraging peer interac- 
tions. An anxious patient may become more agitated by those who are at a more sophisticated knowledge level. Since the data presented tells me that this patient is anxious and it tells me nothing about his peer group, I rate this at a lower priority than I might otherwise.

(7) Peripheral Blood Testing Equipment. Since this person is insulin-requiring it would be best for him to be monitoring his blood glucose levels at home. There is a variety of supplies and equipment for doing these tests. Selection depends upon the person's ability to master the techniques, visual status, including color vision and the financial status.

(8) Clock with a Second Hand. Precision in timing urine and blood tests is important so that a clock with a second hand is essential. For patients who are doing self blood glucose monitoring and are using one of the meters, an automatic timer is frequently built into the equipment.

(9) Series of Patient-Education Classes. Group teaching sessions are valuable in giving broad, general knowledge to people with similar needs and interests. In a group, time and opportunity for individual clarification, support, and instruction is minimal. Our goal with $\mathrm{Mr}$. Benito is to give him information without increasing his anxiety. You have more control over content, can better identify miscommunication, and can individualize the instruction to a greater extent on a one-to-one basis. Group classes are not always the cure-all for lack of knowledge. I would not send him through the entire series but I would send him to selected classes and check his understanding of the information immediately postclass.

(10) Initiation of Visiting Nurse Referral. Obviously only minimal teaching will be done on this admission. Although minimal instruction is indicated and acceptable during this hospitalization, the team has a responsibility to assure continued instruction and health monitoring. A professional who can observe the patient in his own environment, work on a daily and weekly basis with the patient and family, and support the patient and family during initial adjustments is a good solution in this case.

(II) Booklet Regarding Complications of Diabetes. I concur with your decision to delay this information. Information regarding complications should be given to every person with diabetes at some point in their educational process. Now is not the time for this patient. If handled in a positive way, information about complications can motivate people to comply with their regime. However, this patient's anxiety level is already interfering with his ability to learn. I suspect that regardless of its simplicity, the patient may not be ready to hear details on the pathophysiology of diabetes or its complications. (12) Podiatry Referral. There is no evidence of foot problems given in the assessment. Referring the patient to a podiatrist prophylactically might be beneficial. Again the gain should be weighed against the agitation caused by nonessential information during a crisis period. Foot care is very important to every person but with all the concerns that this patient has at the present, now is not the time to overload him with information concerning potential problems.

(13) Discussion Time with a Psychiatrist. Though this resource does not seem indicated at this point, should the patient's anxiety continue at a high level or should the patient voice interest in such a referral, I would definitely utilize this important team member.

(14) Standard 1800-Calorie Preprinted ADA Meal Plan. A standard meal plan is certainly not for this patient or for any patient. To facilitate adherence to a diet, it must be flexible enough to include each person's habits, patterns, likes, and dislikes. If Mr. Benito eats pastas and casserole dishes, then his meal plan should incorporate these in reasonable amounts when possible.

(15) Inject Aid Device. This patient definitely does not require an inject aid. In general these aids are clumsy and cumbersome. If a patient can give his injection, he should do so. An inject aid device does not draw up or give the insulin; it merely puts the needle into the skin. The patient who emotionally cannot tolerate injecting himself may find this useful if other measures fail.

(16) Eyechart. Experience tells me that the blurred vision Mr. Benito has been experiencing with the onset of his diabetes is related to osmotic changes in the lens of his eye as a result of blood glucose fluctuations. While this results in temporary reversible vision changes which are aggravating to the patient, examination using a Jaeger scale or a newspaper will only tell you his present visual status and not what it potentially will be with adequate blood glucose regulation. You should advise him to wait until his blood glucose levels have been stabilized for about six weeks before having refraction examination. Patients are often prescribed new glasses in an attempt to correct this temporary blurred vision when what is needed is a temporary use of a low vision aid, such as a hand magnifier.

(17) Chocolate Candy Bar. While many patients use candy bars to treat hypoglycemia, this is not the preferred treatment. In hypoglycemia the person needs simple carbohydrate which provides quick relief, ie, within ten minutes. A chocolate bar contains a high percentage of fat which takes longer to be utilized and it also adds unneeded calories. Treatment with $10 \mathrm{gm}$ (equivalent to one fruit exchange) of simple carbohydrate such as unsweetened fruit juice, sugar cubes, or lifesavers is the preferred treatment.

The educator stressed, "The success of any education program is measured by how much the patient learns, but it might be interesting to compare our priorities." To determine how your team did compared to my advice, I suggest you score your work.

Using the educator's list as a expert list, score your work in the following way.

1. First Exercise-Individual Scores:

Subtract each item in column I and the corresponding item in column E. (Don't record a $+1-$ sign but merely subtract the smaller number from the larger.) Write the result in column $Z$, then total up the numbers in column $Z$. (This gives the individual versus the expert score.)

2. Second Exercise-Group Decison-Making Score: A. Subtract each item in column $G$ and each corresponding item in column $E$. (Do not record $+1-$ but merely subtract the smaller number from the larger.) Write the result in column $Y$. 
B. Add the numbers in column $Y$ to get the total difference. (This is the group versus the expert score.)

C. Compare the individual scores $(\mathrm{Z})$ with the group decision-making score $(\mathrm{Y})$.

The following conclusions may be drawn regarding the evaluation process just described:

1. The more you know about the key elements involved in a problem, the more successful you are likely to be at finding a workable solution. Therefore a thorough initial assessment and continuing evaluation are critical.

2. A team can usually arrive at a better decision than an individual, especially if the people in the team understand how to work together to share what they know and what they think.

3. Your educational planning is likely to be much more effective if you know which steps to take and how to use each step. If you are skilled at this process you are less likely to commit one of the classic errors, such as generating possible solutions before you identify the problem to be solved. (Note: This may have happened during the Second Exercise: Team Decision-Making, Instructions A and B.) After you properly define both the problem and the goal, you are also in a much better position to decide what information you need to proceed intelligently.

\section{SUMMARY OF STEPS IN PLANNED EDUCATION}

(1) Identify the Educational Needs of the Patient and Family. Considering this patient's social environment, psychological make-up, past experiences, and physical abilities, what does he need? What does he already know? What strengths can you build on? What misconceptions and fears does he harbor?

A. Gather information. Sufficient information is seldom acquired during a single interview or by a single person. This is an ongoing process involving the entire team. B. Identify the problem. Look beyond the symptoms of the problem to find out what is wrong. State the problem carefully as it will influence the direction you take in seeking solutions. Ask the patient to identify his problems. Share the problems you identify with the patient.
(2) Establish Educational Goals for/with Patient and Family. Consider the type of information all patients need to know about a specific illness, then structure the treatment plan so that this patient can accept and carry it out. Ask the patient and family what their goals are. Share your goals with the patient and his family-they must understand and accept the goals if change is to occur.

A. State the basic objective or goal. An objective focuses your thoughts on the real problem, yet is broad enough so that no reasonable solution is overlooked.

B. State the facts, constraints, and assumptions. Facts are statements of things that are known. Constraints are factors which limit the outcome of the project and cannot be changed, and assumptions are applied to factors which can be changed to simplify the problem and make it solvable.

(3) Select Appropriate Educational Methods. Methods should be selected by the team and be specific to each goal. Criteria for selecting educational methods should include (1) effectiveness (extent to which an activity achieves the goal), (2) efficiency (amount of resources used to attain the goal), (3) adequacy (degree to which an educational activity can achieve the goal), and (4) appropriateness (relevancy of the method toward achieving the goal).

A. Generate possible solutions. Be a creative thinker. Don't prejudge ideas as they are generated. Get all the different thoughts you can.

B. Evaluate the possibilities and make a decision. Determine which possible solution is most likely to solve the problem.

C. Analyze. Separate the chosen solution into meaningful elements. Determine and gather the information you need to develop each element.

D. Synthesize. Combine the elements to create a detailed plan.

(4) Evaluate Patient and Family Education. Identify evaluation methods early in the process. Clearly stated goals make evaluation easier. Evaluation of goals must be constant and continuous to prevent treating patients routinely. Is this a method of using staff and patient time to the best advantage? Can the patient perform as adequately with less information? 